\title{
1 Mixing in the food industry: trends and challenges
}

\author{
P.J. Cullen and Colm P. O’Donnell
}

\subsection{Role of mixing}

Mixing is a fundamental unit operation in the chemical, pharmaceutical and food process industries. Mixing increases the homogeneity of a system by reducing non-uniformity or gradients in composition, properties or temperature. Besides the primary objective of homogeneity, secondary objectives of mixing include control of heat and mass transfer rates, reactions and structural changes (Harnby et al. 2001). In food processing applications, additional mixing challenges include sanitary design, complex rheology, desire for continuous processing and the effects of mixing on final product texture and sensory profiles.

The mixing of liquids, solids and gases is one of the most common unit operations in the food industry. Mixing is frequently employed to develop the desired product characteristics such as texture rather than simply ensure product homogeneity. If mixing fails to achieve the required product yield, quality, and organoleptic or functional attributes, production costs may increase significantly.

\subsection{Design criteria for mixing}

As described earlier, mixing is rarely a process with a sole intended effect; a number of physico-chemical processes may occur simultaneously within a mixer. Consequently, numerous mixer designs are proposed to meet these demands (Chapter 5). Although categorisation of mixing equipment is difficult, some mixers are used primarily for either liquids or powders, whereas others are employed for combinations of liquids and powders. Many unique design challenges apply to food mixing as food properties such as texture, flavour, shelf-life and safety must also be considered. The effectiveness of mixing can be assessed only in the context of the quality of the end product.

Within the food industry, relationships established between the quality of mixed products and the operating parameters of mixing are often empirical, and consequently, the design of mixing systems are frequently not based upon well-established scientific principles. Patwardhan and Joshi (1999) indicated an enormous scope for improvement in the efficiency of mixing processes (mixing time per unit power consumption). Mixer design is slowly changing from a complete experimental process to a partially numerical and experimental one. Consequently, the design of new mixing devices is more efficient. On-going demand for improved impeller designs usually comes from the users of industrial mixing equipment when vessels are to be designed for new plants or improvement in the existing design is 
desired for enhancing quality, capacity, process efficiency and energy efficiency. For meeting these objectives, it is imperative that the relationship between the flow pattern and the design objective is understood (Patwardhan \& Joshi 1999).

There is a need to develop and evaluate new in-line mixers to meet the requirements of the food industry, where there is an increasing trend towards high-capacity continuous processes. Irrespective of the type of mixer used, hygienic design and suitability for cleaningin-place are critical. Static mixers may be employed for continuous processing, making them an attractive alternative to stirred tanks, as similar and sometimes better performance can be achieved at lower cost. Motionless mixers, typically, have lower energy consumptions and are of sanitary design due to the absence of moving parts (Thakur et al. 2003).

The energy requirements for food mixing applications can vary significantly. For example, emulsification requires high energy levels, whereas dispersion of delicate particulate matter in shear sensitive liquids requires much lower energy levels. Mechanical damage to food particles in suspension can result from the stirring action of an impeller. It is possible that resultant comminution or attrition of the particles might be such that the overall quality of the product is affected.

Given the wide variety and complexity of mixing tasks, careful design and scale-up studies are required to ensure that effective mixing is achieved in an efficient manner. Mixing scale-up is an empirical process that begins when the first ingredient is chosen, and continues until a successful process is in production (Chapter 6). The challenge is to scale up the equipment and ingredients to an effective size for production, while duplicating the results obtained during development. Failure to adequately consider mixing issues at laboratory or pilot scale may result in significant problems on scale-up. The costs incurred in solving these problems may be significantly more than the cost of investigating and solving mixing problems during the process development phase.

\subsection{Specific challenges in food mixing}

Significant advances in food mixing have been derived from chemical engineering research. Consequently, the complex rheological challenges arising from non-Newtonian food materials and the inclusion of large particles have not been sufficiently investigated. The complex rheology of food products can influence the effectiveness of a given geometry to achieve the desired mixing outcomes (Chapter 3). Food mixing can involve ingredients of different physical properties and quantities. Food materials mixed may range from nanoemulsions, to large particulate suspensions, to highly viscous pastes or dry powders.

The wide variety of particulates with different sizes, shapes and strengths used in the food industry can result in major segregation issues. Producing particulates with similar sizes, agglomerating ingredients, or reducing vibration during transport can all help reduce these segregation problems (Chapter 13).

Also, as food safety is of critical importance in the food industry, mixing processes should not contaminate the product or allow conditions facilitating microbial growth. Consequently, it is important to use appropriate cleaning regimes and equipment with adequate hygienic design (Chapter 13).

\subsubsection{Quality assurance compliance through mixing}

The evolution of the food industry towards higher level processing, increasing consumer expectations and the recent growth of nutraceuticals necessitates a more scientific understanding of mixing within the food industry. New quality assurance risks are emerging 
with mixed food products due to the addition of functional ingredients and increasingly stringent labelling legislation. In 2006 the EU passed Regulation (EC) No. 1925/2006 to regulate the addition of vitamins, minerals and other substances to foods which have a nutritional or physiological effect. The total amount of such additives present in the food may not exceed the regulated maximum levels listed on the label. In 2007 the U.S. Food and Drug Administration (FDA) issued a final rule for current good manufacturing practices (CGMPs) for dietary supplements, requiring that proper controls are in place to ensure such products are processed in a consistent manner, and meet quality standards including purity, strength, and composition. The blurring of the line between the food and pharmaceutical industries is resulting in novel opportunities for each sector coupled with new challenges. Consequently, the food industry can no longer afford to depend upon a 'black box' approach to mixing.

As the food industry is increasingly governed by legislation, it is imperative that unit operations such as mixing are effectively controlled to reduce product variability. The objective is to move from a paradigm of 'testing quality in' to 'building quality in by design'. Fundamental understanding and optimisation of food mixing will facilitate the evolution of the industry towards higher level processing and novel products with added value.

As stated the food industry has adopted its understanding of mixing from other process industries, including chemical and pharmaceutical. For pharmaceuticals, active ingredients are typically dispersed at relatively low concentrations throughout a dispersion media, making the objective of homogeneity difficult. Pharmaceuticals are similar to nutraceuticals or functional foods with regard to the objective of dispersing such active ingredients equally throughout a carrier; however, there are significant differences. Health-promoting properties of foods are not necessarily due to single components, but rather a few or several active ingredients. This creates a significant paradigm shift from the pharmaceutical model, which is based on the efficacy of single agents. Also, pharmaceutical carriers (excipients) are generally not limited by consumer acceptance criteria of taste, nutrition and texture.

\subsubsection{Engineering texture through mixing}

Mixing in the food industry is employed not only to combine multiple ingredients, but also to modify the structure of foods. The unique aspect of the food industry is the development of texture through mixing and the influence of mixing on the sensory characteristics of foods. Complex food structures influenced by mixing include dough, where shear and extensional forces generated by the mixer may be used to develop flour and water into a viscoelastic protein matrix that is capable of retaining the gas produced during proving and baking (Rielly 1997). Other examples of structured fluids produced by mixing include creams and margarines, where the flow field developed in the mixer is used to disperse one liquid phase in another, forming a stable emulsion with the desired rheological and organoleptic properties (Rielly 1997).

Another unique aspect of mixing within the food industry is where gas bubbles are incorporated into liquid or viscoelastic matrices to impart novel and functional properties. Bubble incorporation in processes such as the manufacture of ice creams and chocolate confectionery is now so widely practised that air and gases are increasingly recognised as food ingredients (Chapter 12). In contrast, bubble incorporation-which inevitably accompanies mixing of viscous recipes such as sauces and salad cream-is undesirable, as this can result in inconsistent filling of packages and can also accelerate spoilage. De-aeration or bubble exclusion can be classified as a food mixing operation, as the end product of their exclusion results in a greater level of homogeneity. 
The development of food micro- or nano-emulsions facilitates the addition of novel sensory characteristics to foods (Chapter 9). This can be achieved via a reduced calorific food in which emulsions are designed to give all the sensory properties of high-fat products with lower calorific content. The addition and delivery of micronutrients in this manner is not detectable to the consumer.

\subsection{Advances in the science of mixing}

The food industry must continually seek improvement in process design to increase efficiency and facilitate the development of novel products. Despite the ubiquity of mixing processes and the vast quantities of materials mixed every day, mixing processes are not fully understood scientifically. Although there is broad agreement that mixing is complicated, there is no agreement as to the source of the complications; from a rheology perspective, the constitutive equation is of paramount importance, whereas from a fluid mechanics viewpoint, the complexities of the flow field are of interest (Ottino 1990).

Advances in computational techniques have facilitated a more fundamental understanding of the mixing process for both complex fluids and mixer designs. Computational fluid dynamics (CFD) provides explanations for fluid flow, heat and mass transfer phenomena, potentially leading to better equipment design and process control for food mixing. Although very significant advances have been made over the last few years in the numerical solution of the equations of fluid motion using CFD methods, relatively few studies have been carried out on food mixing. It is likely that CFD modelling will be increasingly employed for optimisation of food processes including mixing.

Perfect mixing is rarely possible; consequently, mixing will be a source of variability within the manufacturing process. There is an increasing trend in the food industry to adopt a process analytical technology (PAT) framework for innovative process manufacturing and quality assurance. This framework facilitates a move from a paradigm of 'testing quality in' post manufacture to 'designing quality in' during manufacture. Recent developments in imaging and monitoring technologies facilitate the mapping of flow within processing vessels, enabling the identification of regions of poor mixing. Sensing technologies such as NIR spectrometry and NIR chemical imaging have been proposed as control systems for determining the optimum mixing time. NIR chemical imaging is an emerging technique that integrates conventional imaging and spectroscopy to attain both spatial and spectral information from an object. Recently, this technology has shown promise for monitoring the blending of pharmaceutical ingredients. Monitoring and control of the mixing processes in the food industry is critical, as incomplete or over-mixing of a product may result in product separation, attrition and undesirable product texture (Chapter 7). In some applications, the effects of mixing can continue well after the mixing action has ceased, and it could be quite some time before the end point is reached. In such situations, on-line monitoring and process control can be very challenging. Monitoring of mixing in many food applications is particularly challenging, especially in processes involving gas inclusion and crystallisation, where the effects of mixing can continue even after agitation has stopped.

\subsection{Book objectives}

This book addresses an identified gap in the literature by providing a dedicated and in-depth reference for mixing processes within the food industry. Although there are a 
number of creditable references covering general mixing, such publications are often biased towards the chemical industry; therefore, topics specific to food mixing and unique food applications are often neglected. This book brings together essential information on the principles and applications of mixing within food processing.

This book covers the underlying principles of mixing, equipment design, novel monitoring techniques and numerical techniques available to advance the scientific understanding of food mixing. Food mixing applications are described in detail. This book will be useful for engineers and scientists who need to specify and select mixing equipment for specific processing applications. It will also assist with the identification and solving of the wide range of mixing problems that occur in industry.

\section{References}

Harnby, M., Edwards, M.F. \& Nienow, A.W. (2001). Mixing in the Process Industries. ButterworthHeinemann, Oxford.

Ottino, J.M. (1990). Mixing, chaotic advection and turbulence. Annual Reviews in Fluid Mechanics, 22, 207-253.

Patwardhan, A.W. \& Joshi, J.B. (1999). Relation between flow pattern and blending in stirred tanks. Industrial and Engineering Chemistry Research, 38, 3131-3143.

Rielly, C.D. (1997). Mixing in food processing. In: Chemical Engineering for the Food Industry (eds P.J. Fryer, D.L. Pyle \& C.D. Rielly). Chapman \& Hall, London.

Thakur, R.K., Vial, C., Nigam, K.D., Nauman, E.B. \& Djelveh, G. (2003). Static mixers in the process industries - a review. Transactions of the Institution of Chemical Engineers, 81, 787-826. 